\title{
Do Copy Number Changes in CACNA2D2, CACNA2D3, and CACNA1D Constitute a Predisposing Risk Factor for Alzheimer's Disease?
}

\author{
Darine Villela ${ }^{1}$, Claudia K. Suemoto ${ }^{2,3}$, Carlos A. Pasqualucci ${ }^{3,4}$, Lea T. Grinberg ${ }^{3,5}$ and \\ Carla Rosenberg ${ }^{1 *}$
}

' Human Genome and Stem-Cell Research Center, Department of Genetics and Evolutionary Biology, Institute of Biosciences, University of São Paulo, São Paulo, Brazil, ${ }^{2}$ Discipline of Geriatrics, Department of Clinical Medicine, School of Medicine, University of São Paulo, São Paulo, Brazil, ${ }^{3}$ Brazilian Aging Brain Study Group - LIM22, Department of Pathology, School of Medicine, University of São Paulo, São Paulo, Brazil, ${ }^{4}$ Department of Pathology, School of Medicine, University of São Paulo, São Paulo, Brazil, ${ }^{5}$ Memory and Aging Center, Department of Neurology, University of California, San Francisco, San Francisco, CA, USA

\section{OPEN ACCESS}

Edited by: Shin Murakami,

Touro University California, USA

Reviewed by:

Ancha Baranova,

George Mason University, USA

Sangkyu Kim,

Tulane University Health Sciences

Center, USA

Anatoliy I. Yashin,

Duke University, USA

${ }^{*}$ Correspondence:

Carla Rosenberg

carlarosenberg@uol.com.br

Specialty section:

This article was submitted to Genetics of Aging,

a section of the journal

Frontiers in Genetics

Received: 23 February 2016 Accepted: 27 May 2016

Published: 14 June 2016

Citation:

Villela $D$, Suemoto $C K$ Pasqualucci CA, Grinberg LT and Rosenberg C (2016) Do Copy

Number Changes in CACNA2D2,

CACNA2D3, and CACNA1D

Constitute a Predisposing Risk Factor

for Alzheimer's Disease?

Front. Genet. 7:107.

doi: 10.3389/fgene.2016.00107
Dysregulation of calcium $\left(\mathrm{Ca}^{2+}\right)$ homeostasis is now being recognized to be a key step in the pathogenesis of Alzheimer's disease (AD). Data from the literature, in particular the association between $\mathrm{AD}$ and polymorphism that interfere with $\mathrm{Ca}^{2+}$ homeostasis indicates the presence of genetic factors in this process; further, presenilins mutations, which are known to cause the familial form of $A D$, are involved in the regulation of intracellular $\mathrm{Ca}^{2+}$ stores. Here, we wish to draw attention to rare DNA copy number variations identified in two subjects with late-onset $A D$ that led to partial or full duplication of genes that encode different subunits of the same type of voltage-gated $\mathrm{Ca}^{2+}$ channels; these duplications of voltage-gated $\mathrm{Ca}^{2+}$ channel genes is consistent with the critical role of calcium signaling in molecular processes underlying memory as has been demonstrated by several studies.

Keywords: Alzheimer's disease, copy number variations, CNVs, array-CGH, $\mathrm{Ca}^{2+}$

It's widely known that $\mathrm{Ca}^{2+}$ represents one of the most important second messengers in the brain and plays an essential role in neuronal development, synaptic transmission and plasticity, besides regulating various metabolic pathways (Striessnig et al., 2006). The regulation of $\mathrm{Ca}^{2+}$ homeostasis in the central nervous system is a very complex process involving proteins localized in the plasma membrane, endoplasmic reticulum (ER), mitochondria, and cytoplasm that together are responsible for maintaining a higher concentration of $\mathrm{Ca}^{2+}$ in the extracellular space (Berridge et al., 2003). Notably, as demonstrated by several studies, a dysregulation in $\mathrm{Ca}^{2+}$ homeostasis is associated with many pathological mechanisms, especially those related with neurodegenerative disorders (Berridge, 2013; Sulzer and Surmeier, 2013).

Based on previous observations that intracellular $\mathrm{Ca}^{2+}$ levels are increased in aging neurons, it has been suggested that a dysregulation of $\mathrm{Ca}^{2+}$ homeostasis could be a key step in the pathogenesis of Alzheimer's disease (AD; Small, 2009). In fact, this is the basis of the calcium hypothesis of AD, which offers a potential correlation between $\beta$-amyloid $(\mathrm{A} \beta)$ toxicity and a progressive decline in memory and neuronal cell death (Fedrizzi and Carafoli, 2011). The central idea is that activation of the amyloidogenic pathway leads to a remodeling of the neuronal $\mathrm{Ca}^{2+}$ signaling, and this remodeling usually appears as upregulated in $\mathrm{AD}$, triggered by either an enhance in the entry of external $\mathrm{Ca}^{2+}$ or a release from internal stores (Berridge, 2010). 
A
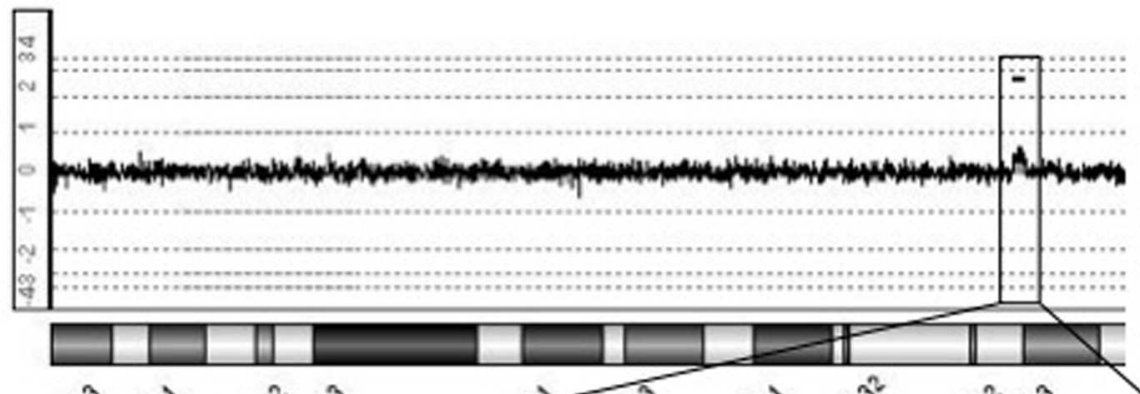

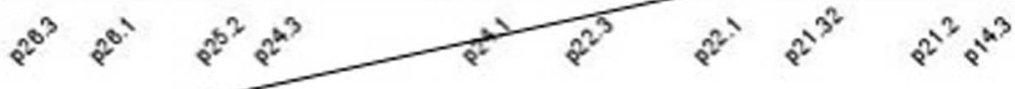

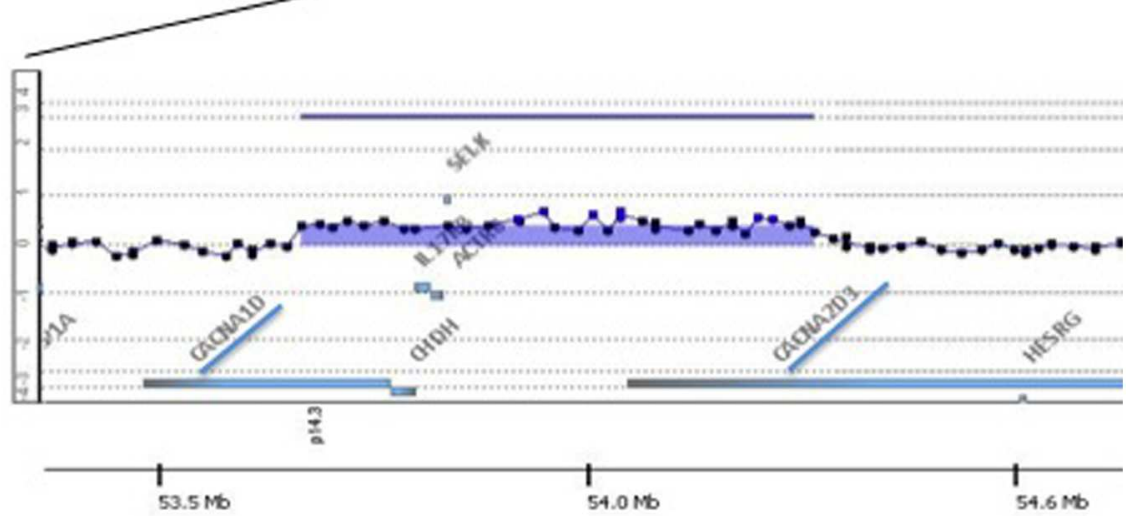

B
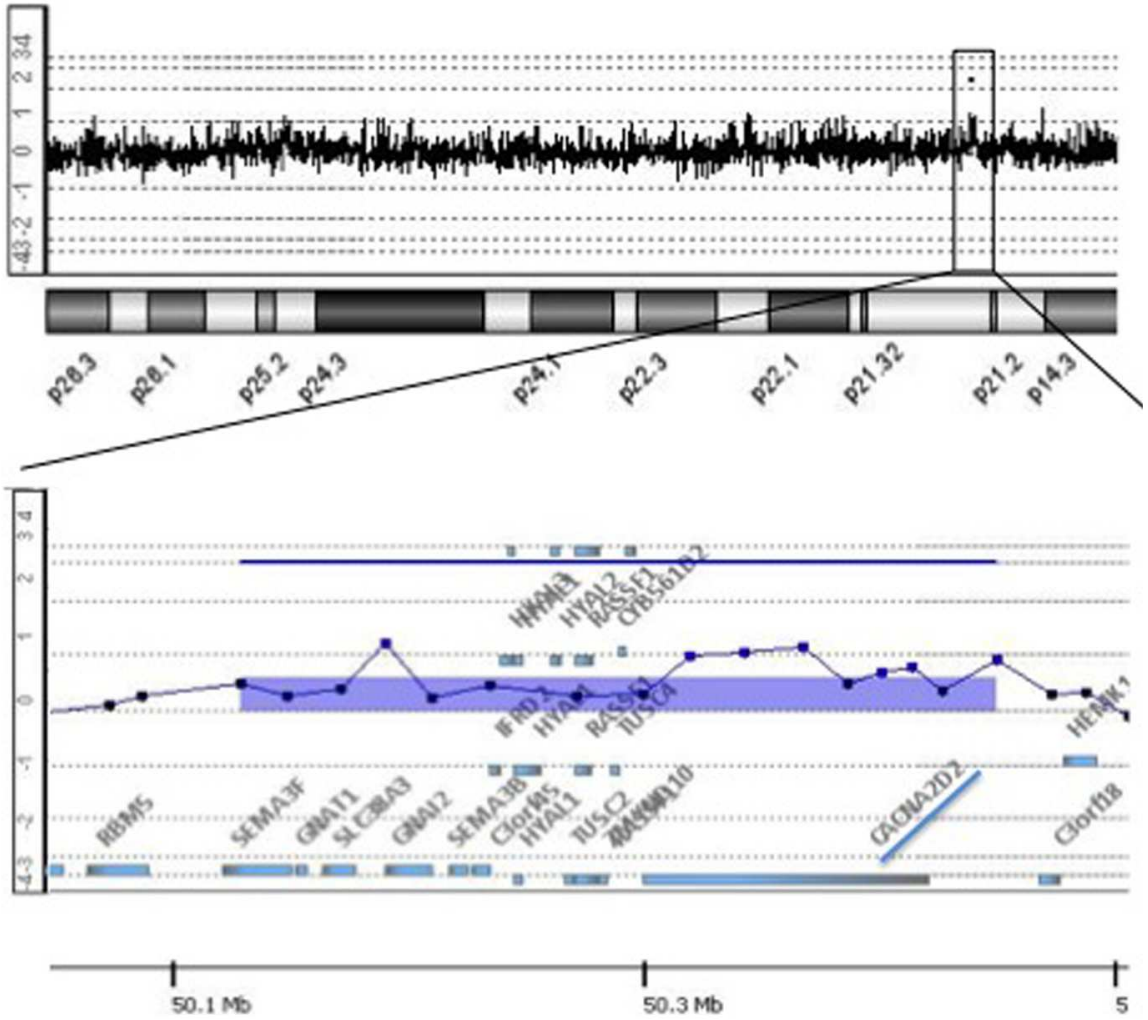

FIGURE 1 | Continued 


\section{FIGURE 1 | Continued}

Rare copy number variations (CNVs) implicated in voltage gate calcium channels detected in two individuals with late-onset Alzheimer's disease. The figure shows array-CGH results where each dot represents a probe. A genomic segment was considered duplicated or deleted when the log 2 ratio of the Test/Reference fluorescent intensities of a given region encompassing at least three probes was above 0.3 or below -0.3, respectively, (Images were extracted from Genomic Workbench software). (A) Top shows array-CGH profile of chromosome 3 of Individual 1; the black box indicates the location of the microduplication on the short arm of the chromosome; underneath a detailed view of the area comprised in the black box at 3p21.1; the image shows a 665 kb duplication containing among other genes, CACNA1D and CACNA2D3, highlighted by a blue trace. (B) Top shows array-CGH profile of chromosome 3 of Individual 2; the black box indicates the location of the microduplication on the short arm of the chromosome; underneath a detailed view of the area comprised in the black box at 3p21.31; the image shows a $300 \mathrm{~kb}$ duplication containing among other genes, CACNA2D2, highlighted by a blue trace.

The $\mathrm{A} \beta$ peptide, present in the extracellular senile plaques, is one of the hallmarks of the disease and its aggregation in oligomers has been found to induce $\mathrm{Ca}^{2+}$ influx into neurons functioning as channels and/or by activating channels in the plasma membrane (Demuro et al., 2010). The A $\beta$ oligomers could form non-selective pores with high conductance for $\mathrm{Ca}^{2+}$ but they could also alter the fluidity of the membrane affecting the activity of several $\mathrm{Ca}^{2+}$ permeable channels, including acetylcholine receptors, glutamate receptors, dopamine receptors, and serotonin receptors (Eckert et al., 2005). On the other hand, within the neurons, $A \beta$ interacts with two types of $\mathrm{Ca}^{2+}$ channels in the ER membrane that regulate calcium release from internal stores: (i) ryanodine receptors (RyRs) and (ii) inositol trisphosphate receptors (IP3Rs); this leads to an increase of their opening and the amount of $\mathrm{Ca}^{2+}$ being released by the ER (Chakroborty et al., 2009). It is relevant to note, though, that data from literature demonstrate that genetic factors may be crucial in the dysregulation of $\mathrm{Ca}^{2+}$ homeostasis in $\mathrm{AD}$. Presenilins mutations, which are known to cause the familial form of $\mathrm{AD}$, have been found to increase the release of intracellular $\mathrm{Ca}^{2+}$ from internal stores (Honarnejad and Herms, 2012; Bezprozvanny, 2013). The activity of the SERCA pump, which is responsible for maintain the $\mathrm{Ca}^{2+}$ concentration gradient across the ER membrane (higher inside) is enhanced by the presenilins (Green et al., 2008). Additionally, there is increasing evidence that presenilins may be leak channel. Tu et al. (2006) reported that the mutated form of presenilin 1 (PS1) reduces the passive leak in neurons resulting in an increase of cytoplasmatic $\mathrm{Ca}^{2+}$.

Of particular interest, it was demonstrated that a polymorphism in CALHM1 $\left(\mathrm{Ca}^{2+}\right.$ homeostasis modulator-1) was significantly associated with the sporadic form of the disease in a case-control study (Dreses-Werringloer et al., 2008). Based on its sequence similarity to the ionotropic glutamate receptor NMDA, it has been proposed that this gene encodes a glycoprotein that is a cerebral $\mathrm{Ca}^{2+}$ channel component, which is localized both in the plasma membrane and ER, and may also function as a leak channel (Dreses-Werringloer et al., 2008). The expression of CALHM1 polymorphic variant reduces $\mathrm{Ca}^{2+}$ entry and this reduced $\mathrm{Ca}^{2+}$ permeability would increase the level of stored $\mathrm{Ca}^{2+}$ by reducing this putative leak pathyway; consequently, a decreased intracelullar $\mathrm{Ca}^{2+}$ levels is observed (Dreses-Werringloer et al., 2008). The authors also suggest a direct impact of CALHM1 on $\mathrm{A} \beta$ production. Although some studies argue against the latter observation, it seems that the problem is the different methods used for analysis of the data (Bertram et al., 2008; Rubio-Moscardo et al., 2013). Besides, even though it seems controversial that in the familial form of $\mathrm{AD}$ an increase of intracellular $\mathrm{Ca}^{2+}$ is observed while in the sporadic form studies report a reduce in intracellular $\mathrm{Ca}^{2+}$ levels, dysregulation of $\mathrm{Ca}^{2+}$ homeostasis in the two forms of $\mathrm{AD}$, as described above, arise by different mechanisms, but what is pretty clear in the literature is that dysregulation of $\mathrm{Ca}^{2+}$ homeostasis is a major feature in $\mathrm{AD}$.

More recently, a multicenter study reported a robust and consistently significant enrichment for genes constituting the calcium signaling pathway, especially those related to the elevation of cytosolic calcium, in independent cohorts of young and elderly participants (Heck et al., 2015). The authors also showed that the same gene set identified in those participants was significantly enriched in a very large case-control study of sporadic $\mathrm{AD}$, making evident that calcium signaling is crucial in human memory processes, both in cognitive health and disease (Heck et al., 2015).

In an investigation about the frequency of DNA copy number variations (CNVs) in a cohort of 46 individual's diagnosed postmortem with late-onset $\mathrm{AD}$ provided by the Brain Bank of the Brazilian Aging Brain Study Group (BBBABSG; Grinberg et al., 2007). We found two cases with rare microduplications partially or fully affecting genes that encode different subunits of the same type of voltage-gated $\mathrm{Ca}^{2+}$ channels, the L-type (L-VGCC). One individual presented a genomic imbalance of $665 \mathrm{~kb}$ at 3p21.1 that incudes, among other genes, CACNA1D and CACNA2D3 (Figure 1A). CACNA1D encodes the subunit $\alpha 1$ (subtype ${ }_{1 D}$ ), which form the ion-conducting pore of the L-VGCC, and CACNA2D3 is responsible for encoding the subunit $\alpha-2 \delta$, that influences the channels' biophysical properties by increasing the current amplitude and regulating the activation and inactivation kinetics of the L-VGCC. On the other hand, the other subject showed a genomic imbalance of $300 \mathrm{~kb}$ at 3p21.31 that encompass the CACNA2D2 gene, which acts similarly to CACNA2D3, i.e., also encodes the subunit $\alpha-2 \delta$ of the L-VGCC (Figure 1B). Both duplications have intragenic breakpoints; depending on the resulting structure of the rearrangement, the partial gene duplications can result in loss of function of the genes involved.

To exclude that these rare CNVs (rare defined as frequency $<0.1 \%$ of population, based on the Database of Genomic Variants - DGV) represent common variants in the Brazilian population, we compile $\mathrm{CNV}$ data from more than 1,300 individuals studied by array-CGH in our laboratory for reasons 
other than dementia, such as cancer predisposition, deafness, congenital abnormalities, and intellectual disability. The rare CNVs documented in this study were never detected in any of these subjects. Additionally, in contrary to the control sample in this study, which consisted in individuals with no anatomophatological signs of $\mathrm{AD}$, this set of more than 1,300 individuals is supposedly similar to the population regarding $\mathrm{AD}$ predisposition factors. In particular, despite some of the other individuals investigated in our casuistic also presented rare CNVs, these two cases are the only ones who showed copy number changes in genes that constitute the calcium signaling pathway. However, we cannot exclude that the remaining 44 individuals may have point mutations in relevant genes of calcium signaling pathway that could not be observed due to a limited resolution of array-CGH.

CNVs are recognized to be a prevalent form of common genetic variation and represent a substantial proportion of total genetic variability in human population. The functional impact of CNVs has been demonstrated at all biological levels, from cellular effects on gene expression to their association with several types of complex traits and genetic diseases (Stankiewicz and Lupski, 2010). To date, copy number changes in genes encoding calcium-signaling molecules have been associated especially with neuronal development disorders, such as autism (Rosenfeld et al., 2010), psychiatric diseases (Lee et al., 2012), and amyotrophic lateral sclerosis.

The present evidence is the first to highlight genomic imbalances in genes that are known to encode important calcium-signaling molecules, and link those CNVs to lateonset $\mathrm{AD}$. Of note, in the manuscript from Heck et al. (2015) it was demonstrated that the same gene family of CACNA1D, CACNA2D2, and CACNA2D3 was found to be significantly enriched in patients with $\mathrm{AD}$, which reinforces the significance of our findings. The present data also contribute adding new mutations in genes of calcium signaling pathway and associate these mutations with $\mathrm{AD}$ since CACNA1D, CACNA1D, CACNA2D2, and CACNA2D3 are not in the list of genes studied by Heck et al. (2015). Thus, considering the importance of L-VGCC in the central nervous system and $\mathrm{Ca}^{2+}$ being such an important regulator of synaptic plasticity, we could suppose that a disruption in CACNA1D, CACNA2D3, and CACNA2D3 caused by CNVs may lead to

\section{REFERENCES}

Berridge, M. J. (2010). Calcium hypothesis of Alzheimer's disease. Pflugers. Arch. 459, 441-449. doi: 10.1007/s00424-009-0736-1

Berridge, M. J. (2013). Dysregulation of neural calcium signaling in Alzheimer disease, bipolar disorder and schizophrenia. Prion 7, 2-13. doi: $10.4161 /$ pri.21767

Berridge, M. J., Bootman, M. D., and Roderick, H. L. (2003). Calcium signalling: dynamics, homeostasis and remodelling. Nat. Rev. Mol. Cell Biol. 4, 517-529. doi: $10.1038 / \mathrm{nrm} 1155$

Bertram, L., Schjeide, B. M., Hooli, B., Mullin, K., Hiltunen, M., Soininen, H., et al. (2008). No association between CALHM1 and Alzheimer's disease risk. Cell 135, 993-994; author reply 994-996. doi: 10.1016/j.cell.2008. 11.030

Bezprozvanny, I. (2013). Presenilins and calcium signaling-systems biology to the rescue. Sci. Signal. 6, e24. doi: 10.1126/scisignal.2004296 a dysregulation in $\mathrm{Ca}^{2+}$ homeostasis and contribute to the pathogenesis of $\mathrm{AD}$. Also, using the Variant Effect Predictor (VEP) tool from Ensemble ${ }^{1}$ to predict the effects of these two microduplications on genes, transcripts, and protein sequence, as well as regulatory regions, we found that the most likely consequence (34\%) is amplification of the amount of transcript. Therefore, this prediction suggests a possible upregulation of $\mathrm{Ca}^{2+}$ signaling, which meets the central idea of the calcium hypothesis of AD (Berridge, 2010). Yet, the crucial question of whether the microduplications showed in this study constitutes a predisposing risk factor for $\mathrm{AD}$ can only be answered by similar investigations in large cohorts, which we hope will be stimulated by this report. Also, we believe that the present evidence may stimulate further functional studies and eventually confirm the impact of these copy number changes in the pathogenesis of $\mathrm{AD}$.

In summary, we wish to draw attention to rare DNA CNVs identified in two subjects with late-onset $\mathrm{AD}$ that led to partial or full duplication of genes that encode different subunits of the same type of voltage-gated $\mathrm{Ca}^{2+}$ channels and point out that the present finding is consistent with the critical role of calcium signaling in molecular processes underlying memory as has been demonstrated by several studies.

\section{AUTHOR CONTRIBUTIONS}

This study was coordinated by CR and DV, as the principal investigator, provided conceptual and technical guidance for all aspects of this study. CS, LG, and CP coordinate the Brain Bank of the Brazilian Aging Study Group and provided all the samples. This commentary was written by DV.

\section{ACKNOWLEDGMENTS}

This work was supported by FAPESP grants CR and CEPIDFAPESP (2009/00898-1; 2013/08028-1), and DV was supported by FAPESP PhD fellowship (2010/15503-0). We thank the Brazilian Aging Brain Study Group for providing DNA samples.

\footnotetext{
${ }^{1}$ http://www.ensembl.org/info/docs/tools/vep/index.html
}

Chakroborty, S., Goussakov, I., Miller, M. B., and Stutzmann, G. E. (2009). Deviant ryanodine receptor-mediated calcium release resets synaptic homeostasis in presymptomatic 3xTg-AD mice. J. Neurosci. 29, 9458-9470. doi: 10.1523/JNEUROSCI.2047-09.2009

Demuro, A., Parker, I., and Stutzmann, G. E. (2010). Calcium signaling and amyloid toxicity in Alzheimer disease. J. Biol. Chem. 285, 12463-12468. doi: 10.1074/jbc.R109.080895

Dreses-Werringloer, U., Lambert, J. C., Vingtdeux, V., Zhao, H., Vais, H., Siebert, A., et al. (2008). A polymorphism in CALHM1 influences Ca2+ homeostasis, Abeta levels, and Alzheimer's disease risk. Cell 133, 1149-1161. doi: $10.1016 /$ j.cell.2008.05.048

Eckert, G. P., Wood, W. G., and Muller, W. E. (2005). Membrane disordering effects of beta-amyloid peptides. Subcell Biochem. 38, 319-337. doi: 10.1007/0387-23226-5_16

Fedrizzi, L., and Carafoli, E. (2011). Ca2+ dysfunction in neurodegenerative disorders: Alzheimer's disease. Biofactors. 37, 189-196. doi: 10.1002/biof.157 
Green, K. N., Demuro, A., Akbari, Y., Hitt, B. D., Smith, I. F., Parker, I., et al. (2008). SERCA pump activity is physiologically regulated by presenilin and regulates amyloid beta production. J. Gen. Physiol. 181, 1107-1116. doi: 10.1085/JGP1322OIA1

Grinberg, L. T., Ferretti, R. E., Farfel, J. M., Leite, R., Pasqualucci, C. A., Rosemberg, S., et al. (2007). Brain bank of the Brazilian aging brain study group - a milestone reached and more than 1,600 collected brains. Cell Tissue Bank. 8, 151-162. doi: 10.1007/s10561-006-9022-z

Heck, A., Fastenrath, M., Coynel, D., Auschra, B., Bickel, H., Freytag, V., et al. (2015). Genetic analysis of association between calcium signaling and hippocampal activation, memory performance in the young and old, and risk for sporadic alzheimer disease. JAMA Psychiatry 72, 1029-1036. doi: 10.1001/jamapsychiatry.2015.1309

Honarnejad, K., and Herms, J. (2012). Presenilins: role in calcium homeostasis. Int. J. Biochem. Cell Biol. 44, 1983-1986. doi: 10.1016/j.biocel.2012.07.019

Lee, K. W., Woon, P. S., Teo, Y. Y., and Sim, K. (2012). Genome wide association studies (GWAS) and copy number variation (CNV) studies of the major psychoses: what have we learnt? Neurosci. Biobehav. Rev. 36, 556-571. doi: 10.1016/j.neubiorev.2011.09.001

Rosenfeld, J. A., Ballif, B. C., Torchia, B. S., Sahoo, T., Ravnan, J. B., Schultz, R., et al. (2010). Copy number variations associated with autism spectrum disorders contribute to a spectrum of neurodevelopmental disorders. Genet. Med. 12, 694-702. doi: 10.1097/GIM.0b013e3181f0c5f3

Rubio-Moscardo, F., Seto-Salvia, N., Pera, M., Bosch-Morato, M., Plata, C., Belbin, O., et al. (2013). Rare variants in calcium homeostasis modulator 1 (CALHM1) found in early onset Alzheimer's disease patients alter calcium homeostasis. PLoS ONE 8:e74203. doi: 10.1371/journal.pone.0074203
Small, D. H. (2009). Dysregulation of calcium homeostasis in Alzheimer's disease. Neurochem. Res. 34, 1824-1829. doi: 10.1007/s11064-009-9960-5

Stankiewicz, P., and Lupski, J. R. (2010). Structural variation in the human genome and its role in disease. Annu. Rev. Med. 61, 437-455. doi: 10.1146/annurev-med100708-204735

Striessnig, J., Koschak, A., Sinnegger-Brauns, M. J., Hetzenauer, A., Nguyen, N. K., Busquet, P., et al. (2006). Role of voltage-gated L-type Ca2+ channel isoforms for brain function. Biochem. Soc. Trans. 34, 903-909. doi: 10.1042/BST03 40903

Sulzer, D., and Surmeier, D. J. (2013). Neuronal vulnerability, pathogenesis, and Parkinson's disease. Mov. Disord. 28, 715-724. doi: 10.1002/mds.25187

Tu, H., Nelson, O., Bezprozvanny, A., Wang, Z., Lee, S. F., Hao, Y. H., et al. (2006). Presenilins form ER Ca2+ leak channels, a function disrupted by familial Alzheimer's disease-linked mutations. Cell 126, 981-993. doi: 10.1016/j.cell.2006.06.059

Conflict of Interest Statement: The authors declare that the research was conducted in the absence of any commercial or financial relationships that could be construed as a potential conflict of interest.

Copyright (c) 2016 Villela, Suemoto, Pasqualucci, Grinberg and Rosenberg. This is an open-access article distributed under the terms of the Creative Commons Attribution License (CC BY). The use, distribution or reproduction in other forums is permitted, provided the original author(s) or licensor are credited and that the original publication in this journal is cited, in accordance with accepted academic practice. No use, distribution or reproduction is permitted which does not comply with these terms. 